\title{
Relationship between left ventricular diastolic dyssynchrony and systolic dyssynchrony in hypertrophic cardiomyopathy by single-cardiac real-time three-dimensional ultrasonography
}

\author{
BING XING ${ }^{1 *}$, AILIN CAO ${ }^{2 *}$, WEI SUN ${ }^{1}$ and XIYAN HOU ${ }^{3}$ \\ ${ }^{1}$ Department of Cardiology, Jining No. 1 People's Hospital, Jining, Shandong 273200; Departments of ${ }^{2}$ Cardiology \\ and ${ }^{3}$ Emergency, Affiliated Hospital of Jining Medical University, Jining, Shandong 272029, P.R. China
}

Received November 13, 2017; Accepted January 9, 2018

DOI: $10.3892 /$ etm.2018.5872

\begin{abstract}
The relationship between left ventricular diastolic and systolic dyssynchrony in hypertrophic cardiomyopathy (HCM) was investigated by single-cardiac real-time threedimensional ultrasonography. A total of 52 patients with HCM were selected in Jining No. 1 People's Hospital from July 2016 to June 2017. Additionally, a total of 52 healthy people were selected to serve as the control group. All participants received real-time two- and three-dimensional ultrasonography to evaluate left ventricular morphology, function and systolic and diastolic function. The relevant parameters included left ventricular end-diastolic volume (LVEDV), left ventricular end-systolic volume (LVESV), left ventricular ejection fraction (LVEF), end-systolic/diastolic sphericity index (ESSI/EDSI), systolic dyssynchrony index (SDI), diastolic dyssynchrony index (DDI), dispersion end systole (DISPES), diastolic dyssynchrony index-late (DDI-late) and dispersion end diastole (DISPED-late). The LVEF of observation group was significantly lower than that of the control group, while LVEDV, LVESV, E/A and E/Ea were significantly higher than those in control group $(\mathrm{P}<0.05)$; EDSI, DDI-late and DISPED-late were significantly higher in observation than in control group $(\mathrm{P}<0.05)$; ESSI, SDI and DISPES in observation were significantly higher than those in control group $(\mathrm{P}<0.05)$; The 16-segment time-volume curve of observation group was disordered without synchronization, while the curve of control group was regular and smooth with synchronization; Pearson's
\end{abstract}

Correspondence to: Dr Xiyan Hou, Department of Emergency, Affiliated Hospital of Jining Medical University, 89 Guhuai Road, Jining, Shandong 272029, P.R. China

E-mail: neolnnn@163.com

*Contributed equally

Key words: three-dimensional ultrasonography, hypertrophic cardiomyopathy, diastolic dyssynchrony, systolic dyssynchrony, correlation correlation analysis showed that SDI and DDI were positively correlated $(\mathrm{P}<0.05)$. In conclusion, three-dimensional ultrasonography can be used to effectively evaluate left ventricular diastolic and systolic dyssynchrony in HCM. The severity of diastolic is positively correlated with systolic dyssynchrony.

\section{Introduction}

Hypertrophic cardiomyopathy (HCM) is a common sarcomere gene mutation-caused clinical hereditary heart disease mainly characterized by autosomal dominant inheritance and asymmetric hypertrophy in the left ventricle and interventricular septum (1). HCM can be expressed as chest tightness, syncope, chest pain, palpitations and dyspnoea (2). The left ventricular of HCM patients usually show irregular geometry structure, and the unique structure and function can lead to the reduced left ventricular and stroke volume. Pathological manifestations include myocardial hypertrophy and local fibrosis, and myocardial fiber was disorderly arranged, resulting in lower left ventricular compliance and diastolic dysfunction (3). More than $60 \%$ of HCM patients showed familial hereditary, while sporadic only accounted for about $30 \%$. Left ventricular systolic and diastolic function plays an important role in the diagnosis, treatment and prognosis evaluation of HCM. Although two-dimensional echocardiography can provide some information, measurement error is large, resulting in poor accuracy and repeatability. Accurate and effective detection of left ventricular diastolic/systolic function in patients with HCM is important for the treatment and prognosis of this disease. Real-time three-dimensional ultrasound is a new imaging technique, compared with the traditional two-dimensional ultrasound, single-hearted real-time three-dimensional ultrasound can obtain full volume and seamless images, so as to more intuitively and accurately evaluate ventricular diastolic/systolic function of HCM patients, and its repeatability is satisfactory, and can serve as a complement to two-dimensional ultrasound $(4,5)$. At present, studies on the relationship between left ventricular diastolic and systolic dyssynchrony are still lacking. In the present study, singlecardiac real-time three-dimensional ultrasonography was performed for all the patients to investigate the relationship 
Table I. General information of patients.

\begin{tabular}{lcccr}
\hline & \multicolumn{2}{c}{ Groups } & & \\
\cline { 2 - 3 } Items & Observation $\mathrm{n}=52$ & Control $\mathrm{n}=52$ & $\mathrm{t} / \chi^{2}$ & P-value \\
\hline Age (years) & $30-78$ & $30-75$ & & \\
Sex (male/female) & $29 / 23$ & $27 / 25$ & 0.039 & 0.844 \\
Average age (years) & $52.76 \pm 6.45$ & $53.15 \pm 7.13$ & 0.293 & 0.770 \\
BMI $\left(\mathrm{kg} / \mathrm{m}^{2}\right)$ & $23.73 \pm 2.14$ & $24.07 \pm 1.86$ & 0.865 & 0.389 \\
Cultural level & $11(21.16)$ & $12(23.08)$ & 0.168 & 0.919 \\
Junior high school and below & $21(40.38)$ & $19(36.54)$ & & \\
High school and secondary school & $20(38.46)$ & $21(40.38)$ & & \\
College and above & & & & \\
\hline
\end{tabular}

BMI, body mass index.

between left ventricular diastolic and systolic dyssynchrony in HCM to provide references for the treatment of HCM.

\section{Materials and methods}

General information. A total of 52 patients with HCM were selected from July 2016 to June 2017 to serve as observation group. Inclusion criteria: i) With clinical manifestations and echocardiography results in line with HCM diagnostic criteria (6); ii) with stage III diastolic dysfunction according to the diagnostic criteria established by American Society of Echocardiography (ASE); and iii) patients signed informed consent. Exclusion criteria for the study were: i) Received surgery in recent 6 months; and ii) complicated with hypertension, pulmonary heart disease and malignant tumor. A total of 52 cases of healthy people were selected in the same period to serve as the control group. This study was reviewed and approved by the Ethics Committee of Jining No. 1 People's Hospital (Jining, China). Patients provided written informed consent. There was no significant difference in general information between the two groups $(\mathrm{P}>0.05)$ (Table I).

Methods. ACUSON SC2000 ultrasonic diagnostic apparatus (Siemens, Erlangen, GER) was used. 4Z1c full volume probe frequency: $2.8 \mathrm{MHz}$. Scanning angle: $90^{\circ} \times 90^{\circ}$, Scanning depth: $15-16 \mathrm{~cm}$, volume fraction $\geq 12$ frame $/ \mathrm{sec}$. The left lateral position was taken. Conventional two-dimensional ultrasonography was used to measure left ventricular ejection fraction (LVEF), left ventricular end-diastolic volume (LVEDV) and left ventricular end-systolic volume (LVESV) 3 times, and average values were calculated. ECG was connected to record the electrocardiogram and collect the three-dimensional images of three complete cardiac cycles at the end of the breath. Full volume images of the heart and its corresponding three-plane cutting images were collected. The data were analyzed by LVA analysis software, and left ventricle was divided into 16 segments. Related parameters including LVEDV, LVESV, LVEF, end-systolic/diastolic sphericity index (ESSI/EDSI), systolic dyssynchrony index (SDI), diastolic dyssynchrony index (DDI), dispersion end systole (DISPES), diastolic dyssynchrony index-late (DDI-late) and dispersion end diastole (DISPED-late) were obtained. The segment volume-time curve was also obtained.

Evaluation criteria. Patient's diastolic functions were determined according to the criteria established by ASE: Level I: $\mathrm{E} / \mathrm{A}$ ratio $<0.8$, E peak deceleration time $(\mathrm{DT})>200 \mathrm{~ms}, \mathrm{E} / \mathrm{EA}$ ratio $\leq 8$, slightly damaged diastolic function; Level II: $>0.8$ $\mathrm{E} / \mathrm{A}$ ratio $<1.5,>160 \mathrm{DT} \leq 200 \mathrm{~ms}, \geq 9 \mathrm{E} / \mathrm{EA}$ ratio $<12$, false normal diastolic function; and Level III: $\geq 2$ E/A ratio, DT $>160 \mathrm{~ms}, \leq 13 \mathrm{E} / \mathrm{EA}$ ratio, restrictive filling (7).

During real-time three-dimensional ultrasound examination, the left ventricle was divided into 16 segments, and the diastolic volume-time curve of those 16 segments was obtained automatically by software. LVEF, LVEDV, LVESV, EDSI, DDI-late, DISPED-late, ESSI, SDI and DISPES were determined.

Statistical analysis. The data were processed using SPSS19.0 (SPSS, Inc., Chicago, IL, USA) software. Measurement data were expressed as mean \pm standard deviation, and processed by t-test. Enumeration data were expressed as rate, and processed by $\chi^{2}$ test. Correlation analyses were performed using Pearson's correlation coefficient analysis. $\mathrm{P}<0.05$ was considered to indicate a statistically significant difference.

\section{Results}

Comparison of results of two- and three-dimensional ultrasonography. Results of left ventricular function tests in 52 patients with HCM showed that the values of LVEF, LVEDV and LVESV in two-dimensional ultrasonography were significantly higher than those in three-dimensional ultrasonography $(\mathrm{P}<0.05)$ (Table II).

HCM morphological characteristics. Wall thickness of 16 segments of the two groups was measured and results showed that the hypertrophy of HCM was irregular, and left ventricular hypertrophy could occur in any part, such as anterior wall apex, lateral wall apex and left ventricular apex hypertrophy. In 52 HCM patients, 43 cases $(82.69 \%)$ were unsymmetrical hypertrophy of interventricular septum, 
Table II. Comparison of results of two-dimensional and three-dimensional ultrasonography.

\begin{tabular}{|c|c|c|c|c|}
\hline Methods & Cases & $\operatorname{LVEF}(\%)$ & LVEDV (ml) & LVESV (ml) \\
\hline Two-dimensional ultrasonography & 52 & $59.52 \pm 3.42$ & $104.97 \pm 3.84$ & $50.76 \pm 3.46$ \\
\hline Three-dimensional ultrasonography & 52 & $53.78 \pm 3.56$ & $99.13 \pm 3.86$ & $43.87 \pm 3.27$ \\
\hline t-test & & 8.385 & 7.735 & 10.436 \\
\hline P-value & & $<0.001$ & $<0.001$ & $<0.001$ \\
\hline
\end{tabular}

LVEF, left ventricular ejection fraction; LVEDV, left ventricular end-diastolic volume; LVESV, left ventricular end-systolic volume.

Table III. Comparison of cardiac function between the two groups of patients.

\begin{tabular}{|c|c|c|c|c|c|c|}
\hline Groups & Cases & LVEF (\%) & LVEDV (ml) & LVESV (ml) & $\mathrm{E} / \mathrm{A}$ & $\mathrm{E} / \mathrm{Ea}$ \\
\hline Control & 52 & $62.73 \pm 3.68$ & $91.97 \pm 3.64$ & $36.76 \pm 3.18$ & $1.35 \pm 0.23$ & $7.35 \pm 1.16$ \\
\hline Observation & 52 & $53.78 \pm 3.56$ & $99.13 \pm 3.86$ & $43.87 \pm 3.27$ & $2.87 \pm 0.35$ & $13.87 \pm 1.23$ \\
\hline t-test & & 12.605 & 9.732 & 11.240 & 26.172 & 27.809 \\
\hline P-value & & $<0.001$ & $<0.001$ & $<0.001$ & $<0.001$ & $<0.001$ \\
\hline
\end{tabular}

LVEF, left ventricular ejection fraction; LVEDV, left ventricular end-diastolic volume; LVESV, left ventricular end-systolic volume.

Table IV. Comparison of left ventricular diastolic function between two groups (mean $\pm \mathrm{SD})$.

\begin{tabular}{lcccc}
\hline Groups & Cases & EDSI & DDI-late & DISPED-late \\
\hline Control & 52 & $41.52 \pm 3.19$ & $5.18 \pm 0.78$ & $21.25 \pm 1.16$ \\
Observation & 52 & $50.64 \pm 3.73$ & $7.89 \pm 0.95$ & $27.64 \pm 1.58$ \\
t-test & & 13.399 & 15.898 & 23.508 \\
P-value & & $<0.001$ & $<0.001$ & $<0.001$ \\
\hline
\end{tabular}

EDSI, end-diastolic sphericity index; DDI-late, diastolic dyssynchrony index-late; DISPED-late, dispersion end diastole. SD, standard deviation.

$6(11.54 \%)$ were apical hypertrophy and 3 were homogeneous hypertrophy $(5.77 \%)$.

Comparison of cardiac function, left ventricular diastolic function, left ventricular systolic function, diastolic volume-time curve of 16 segments between two groups. LVEF of observation was significantly lower than that of control group, while LVEDV, LVESV, E/A and E/Ea were significantly higher in observation than in control group $(\mathrm{P}<0.05)$ (Table III). EDSI, DDI-late and DISPED-late were significantly higher in the observation than in the control group $(\mathrm{P}<0.05)$ (Table IV). ESSI, SDI and DISPES in observation were significantly higher than those in the control group $(\mathrm{P}<0.05)$ (Table V). The 16-segment time-volume curve of observation group was disordered without synchronization (Fig. 1), while the curve of control group was regular and smooth with synchronization (Fig. 2).

Correlation analysis between left ventricular diastolic and systolic dyssynchrony. Pearson's correlation coefficient
Table V. Comparison of left ventricular systolic function of two groups of patients (mean $\pm \mathrm{SD}$ ).

\begin{tabular}{lcccc}
\hline Groups & Cases & ESSI & SDI & DISPES \\
\hline Control & 52 & $35.54 \pm 3.08$ & $5.36 \pm 1.29$ & $16.78 \pm 3.09$ \\
Observation & 52 & $41.68 \pm 3.27$ & $8.89 \pm 1.64$ & $32.82 \pm 3.53$ \\
t-test & & 9.856 & 12.200 & 24.655 \\
P-value & & $<0.001$ & $<0.001$ & $<0.001$ \\
\hline
\end{tabular}

ESSI, end-systolic sphericity index; SDI, systolic dyssynchrony index; DISPES, dispersion end systole. SD, stardard deviation.

analysis showed that SDI and DDI were positively correlated $(r=0.413, \mathrm{P}<0.05)$ (Fig. 3).

\section{Discussion}

HCM is a hereditary cardiomyopathy, which is caused by mutations in the gene encoding the sarcoma structural protein gene. HCM is mainly expressed as the left ventricular wall asymmetric thickening, and the prevalence rate is approximately $0.2 \%(8)$. The most important pathophysiological change of HCM is the left ventricular diastolic dysfunction, resulting in increased left ventricular filling pressure, then blood will be transferred through the left atrial to pulmonary vein to cause high pulmonary blood flow. Patients usually show symptoms such as shortness of breath and chest tightness and severe symptoms can cause sudden death. Cardiac death and malignant arrhythmia and other events are also common, seriously affecting the quality of life of patients $(9,10)$. HCM is mainly affected by genetic factors, and a variety of internal and external environmental factors 


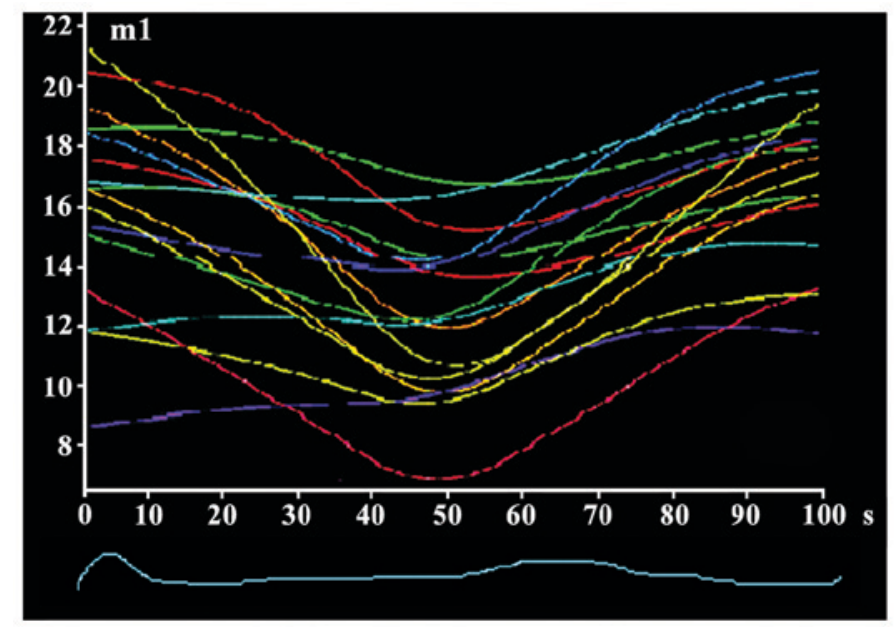

Figure 1. Comparison of diastolic volume-time curve of 16 segments of the observation group.

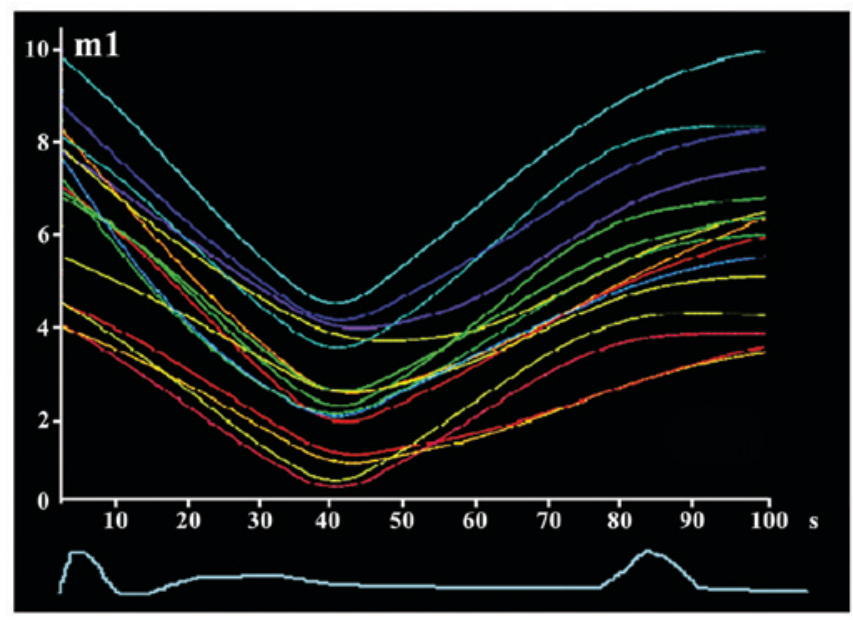

Figure 2. Comparison of diastolic volume-time curve of 16 segments of the control group.

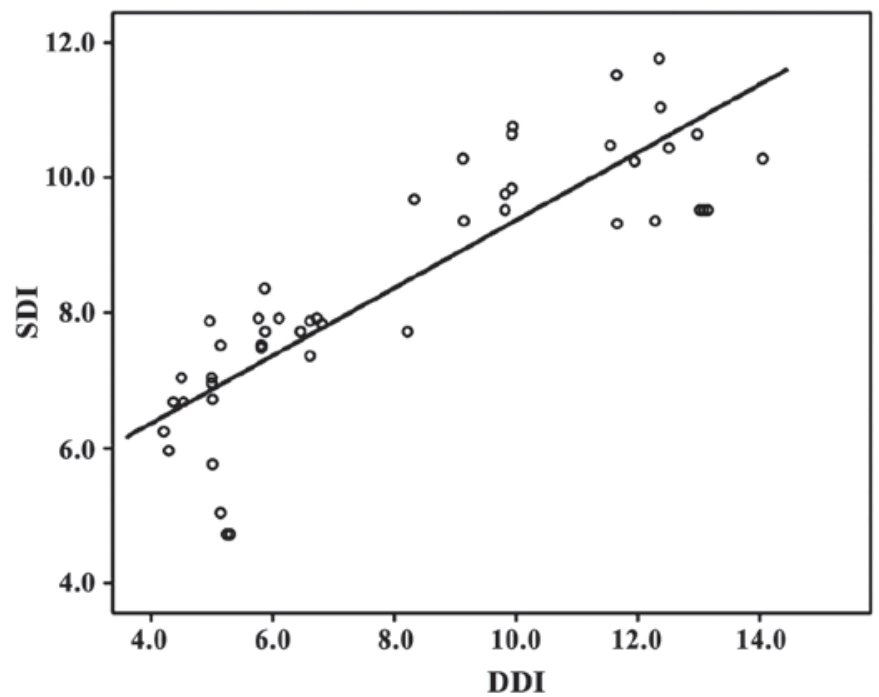

Figure 3. Correlation analysis between left ventricular diastolic and systolic dyssynchrony. Pearson's correlation coefficient analysis showed that SDI and DDI were positively correlated. SDI, systolic dyssynchrony index; DDI, diastolic dyssynchrony index.

can also contribute to the phenotypes (11). The pathogenesis of diastolic dysfunction of HCM patients is complex with the involvement of myocardial fibrosis, ventricular wall diastolic dyssynchrony, energy metabolism, decreased ventricular wall compliance and ventricular wall hypertrophy and other factors (12). 
In the 1970s, three-dimensional imaging of the heart was first applied, and three-dimensional ultrasound imaging was developed from static to dynamic, and even in real time, and was widely used clinically (13). The results of the present study showed that LVEF in observation was significantly lower than that in control group, while LVEDV, LVESV, E/A and E/Ea were significantly higher in observation than those in the control group $(\mathrm{P}<0.05)$. This is because the cardiac hypertrophy, myocardial fiber abnormalities and low ventricular compliance occured in HCM patients, in addition, ventricular wall of HCM patients is harder than that of normal people, and wall tension is also higer, leading to the increased left ventricular load, resulting in left ventricular enlargement. Thus, LVEDV and LVESV of HCM patients are significantly higher than those of normal people (14). Myocardial compliance and relaxation ability of HCM patients were reduced, leading to the limited early ventricular diastolic filling and increased left atrial pressure, and thus E/A and E/Ea ratio were increased (15).

Compared with two-dimensional, real-time threedimensional ultrasound can more precisely and accurately reflect the local changes in the heart, it can also solve the overreliance of two-dimensional ultrasound on geometric model assumptions (16). Single-cardiac real-time three-dimensional ultrasonography can be a perfect solution for the problem of splicing in multi-cardiac cycles three-dimensional ultrasound, it also offers the time needed for image acquisition and data analysis, and the left ventricular volume can also be directly evaluated (17). Results of this study showed that EDSI, DDI-late and DISPED-late were significantly higher in observation than in control group, and ESSI, SDI and DISPES in observation were also significantly higher than those in control group $(\mathrm{P}<0.05)$. Cardiac activity of the normal is expressed by the orderly systolic and diastolic activities of atria and ventricles. Real-time three-dimensional echocardiography showed that the left ventricular 16 segments of the control reached the minimum systolic volume at the same time point of the cardiac cycle, and the volume-time curve showed a smooth trend and the synchronization was good, while mechanical delay was observed in observation group with the most important manifestation of ventricular systolic and diastolic dyssynchrony, therefore, the time points of the 16 segments reached the minimum systolic volume will be different, so the volume-time curve showed irregularity and loss of synchronization. DDI was obtained from the analysis using real-time three-dimensional ultrasonic software to effectively evaluate left ventricular diastolic function. Increased DDI indicate the diastolic dysfunction in HCM patients, while increased SDI is associated with varying degrees of systolic dysfunction (18).

Pearson's correlation coefficient analysis showed that SDI was positively correlated with DDI $(\mathrm{r}=0.413, \mathrm{P}<0.05)$. This is because HCM patients usually had coexistence of left ventricular diastolic and systolic dysfunction. With the progress of HCM disease, myocardial fibrosis will gradually occur, thus affecting the normal metabolism of myocardial energy, increased diastolic dysfunction and DDI, so left ventricular diastolic dyssynchrony will happen, which in turn leads to increased SDI $(19,20)$. SDI will increase with the increase in DDI, and the two are positive correlated, indicating the coexisting of left ventricular diastolic and systolic dyssynchrony in HCM patients.
Collectively, the use of single-cardiac real-time threedimensional ultrasound examination of HCM patients can effectively shorten the image acquisition and analysis time. This technique can be used to intuitively and accurately reflect the left ventricular diastolic and systolic dyssynchrony in HCM patients to assist clinical diagnosis and treatment. This study is limited by the small sample size, future studies with larger sample size are needed to confirm the conclusions in the present study.

\section{Acknowledgements}

Not applicable.

\section{Funding}

No funding was received.

\section{Available of data and materials}

The datasets used and/or analyzed during the present study are available from the corresponding author on reasonable request.

\section{Authors' contributions}

BX drafted this manuscript. BX and AC were mainly devoted on collecting and interpreting the data. XH revised it critically for important intellectual content. WS and XH were responsible for the conception and design of the study. All authors read and approved the final manuscript.

\section{Consent for publication}

This study was reviewed and approved by the Ethics Committee of Jining No. 1 People's Hospital (Jining, China). Patients provided written informed consent.

\section{Competing interests}

Not applicable.

\section{Competing interests}

The authors declare that they have no competing interests.

\section{References}

1. Semsarian C, Ingles J, Maron MS and Maron BJ: New perspectives on the prevalence of hypertrophic cardiomyopathy. J Am Coll Cardiol 65: 1249-1254, 2015.

2. Maron BJ, Rowin EJ, Casey SA, Link MS, Lesser JR, Chan RH, Garberich RF, Udelson JE and Maron MS: Hypertrophic cardiomyopathy in adulthood associated with low cardiovascular mortality with contemporary management strategies. J Am Coll Cardiol 65: 1915-1928, 2015.

3. Elliott PM, Anastasakis A, Borger MA, Borggrefe M, Cecchi F, Charron P, Hagege AA, Lafont A, Limongelli G, Mahrholdt H, et al: Authors/Task Force members: 2014 ESC Guidelines on diagnosis and management of hypertrophic cardiomyopathy: The Task Force for the diagnosis and management of hypertrophic cardiomyopathy of the European Society of Cardiology (ESC). Eur Heart J 35: 2733-2779, 2014.

4. Wasilewska M, Gardziejczyk W and Gierasimiuk P: Evaluation of skid resistance using CTM, DFT and SRT-3 devices. Transportat Res Proced 14: 3050-3059, 2016. 
5. Cai Q and Ahmad M: Left ventricular dyssynchrony by three-dimensional echocardiography: Current understanding and potential future clinical applications. Echocardiography 32: 1299-1306, 2015.

6. Kalsi KK, Smolenski RT, Pritchard RD, Khaghani A, Seymour AM and Yacoub MH: Energetics and function of the failing human heart with dilated or hypertrophic cardiomyopathy. Eur J Clin Invest 29: 469-477, 1999.

7. Dubourg O, Mansencal N and Charron P: Recommendations for the diagnosis and management of hypertrophic cardiomyopathy in 2014. Arch Cardiovasc Dis 108: 151-155, 2015.

8. Alfares AA, Kelly MA, McDermott G, Funke BH, Lebo MS, Baxter SB, Shen J, McLaughlin HM, Clark EH, Babb LJ, et al: CORRIGENDUM: Results of clinical genetic testing of 2,912 probands with hypertrophic cardiomyopathy: Expanded panels offer limited additional sensitivity. Genet Med 17: 319, 2015

9. Cardim N, Galderisi M, Edvardsen T, Plein S, Popescu BA, D'Andrea A, Bruder O, Cosyns B, Davin L, Donal E, et al: Role of multimodality cardiac imaging in the management of patients with hypertrophic cardiomyopathy: An expert consensus of the European Association of Cardiovascular Imaging endorsed by the Saudi Heart Association. Eur Heart J Cardiovasc Imaging 16: $280,2015$.

10. Hensley N, Dietrich J, Nyhan D, Mitter N, Yee MS and Brady M: Hypertrophic cardiomyopathy: A review. Anesth Analg 120: 554-569, 2015.

11. Nomura A, Konno T, Fujita T, Tanaka Y, Nagata Y, Tsuda T, Hodatsu A, Sakata K, Nakamura H, Kawashiri MA, et al: Fragmented QRS predicts heart failure progression in patients with hypertrophic cardiomyopathy. Circ J 79: 136-143, 2015.

12. Ho CY, Lakdawala NK, Cirino AL, Lipshultz SE, Sparks E, Abbasi SA, Kwong RY, Antman EM, Semsarian C, González A, et al: Diltiazem treatment for pre-clinical hypertrophic cardiomyopathy sarcomere mutation carriers: A pilot randomized trial to modify disease expression. JACC Heart Fail 3: 180-188, 2015.

13. Zhu M, Ashraf M, Zhang Z, Streiff C, Shimada E, Kimura S, Schaller T, Song X and Sahn DJ: Real time three-dimensional echocardiographic evaluations of fetal left ventricular stroke volume, mass, and myocardial strain: In vitro and in vivo experimental study. Echocardiography 32: 1697-1706, 2015.

14. Maron BJ, Casey SA, Chan RH, Garberich RF, Rowin EJ and Maron MS: Independent assessment of the European Society of Cardiology sudden death risk model for hypertrophic cardiomyopathy. Am J Cardiol 116: 757-764, 2015.
15. Patel P, Dhillon A, Popovic ZB, Smedira NG, Rizzo J, Thamilarasan M, Agler D, Lytle BW, Lever HM and Desai MY: Left ventricular outflow tract obstruction in hypertrophic cardiomyopathy patients without severe septal hypertrophy: Implications of mitral valve and papillary muscle abnormalities assessed using cardiac magnetic resonance and echocardiography. Circ Cardiovasc Imaging 8: e003132, 2015.

16. Lu KJ, Chen JX, Profitis K, Kearney LG, DeSilva D, Smith G, Ord M, Harberts S, Calafiore P, Jones E, et al: Right ventricular global longitudinal strain is an independent predictor of right ventricular function: A multimodality study of cardiac magnetic resonance imaging, real time three-dimensional echocardiography and speckle tracking echocardiography. Echocardiography 32: 966-974, 2015.

17. Elsayed M, Hsiung MC, Meggo-Quiroz LD, Elguindy M, Uygur B, Tandon R, Guvenc T, Keser N, Vural MG, Bulur S, et al: Incremental value of live/real time three-dimensional over two-dimensional transesophageal echocardiography in the assessment of atrial septal pouch. Echocardiography 32: $1858-1867,2015$

18. Wada Y, Aiba T, Matsuyama TA, Nakajima I, Ishibashi K, Miyamoto K, Yamada Y, Okamura H, Noda T, Satomi K, et al: Clinical and pathological impact of tissue fibrosis on lethal arrhythmic events in hypertrophic cardiomyopathy patients with impaired systolic function. Circ J 79: 1733-1741, 2015.

19. Zhao B, Wang S, Chen J, Ji Y, Wang J, Tian X and Zhi G: Echocardiographic characterization of hypertrophic cardiomyopathy in Chinese patients with myosin-binding protein $\mathrm{C} 3$ mutations. Exp Ther Med 13: 995-1002, 2017.

20. Parthiban A, Li L, Kindel SJ, Shirali G, Roessner B, Marshall J, Schuster A, Klas B, Danford DA and Kutty S: Mechanical dyssynchrony and abnormal regional strain promote erroneous measurement of systolic function in pediatric heart transplantation. J Am Soc Echocardiogr 28: 1161-1170, 2015.

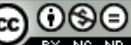

This work is licensed under a Creative Commons Attribution-NonCommercial-NoDerivatives 4.0 International (CC BY-NC-ND 4.0) License. 